\title{
An Evidence Based Approach to Sports Concussion: Confronting the Availability Cascade
}

\author{
William B. Barr
}

Received: 10 November 2013 / Accepted: 11 November 2013 /Published online: 27 November 2013

(C) Springer Science+Business Media New York 2013

This special issue of Neuropsychology Review consists of six articles covering the topic of sport related concussion (SRC) prepared by a distinguished panel of scientists. The past 20 years have been marked by a steady increase in the public's focus on brain injury in athletes, fueled primarily by media accounts of extraordinary outcomes, including prolonged symptoms or untimely deaths, in professional athletes sustaining concussions in football, ice hockey, and other contact sports. Over the same time period, there has also been an unprecedented level of scientific investigation on SRC. The articles contained in this issue provide contemporary evidence-based reviews of where the literature currently resides on a range of topics related to SRC, including epidemiology, acute effects, methods of assessment, and long-term outcome.

A common theme of several of these paper is that there has been a divergence of media accounts of SRC in relation to the reviewed segment of existing scientific and medical findings. Although results of controlled investigations of SRC and guidelines for its management indicate that the vast majority of athletes will demonstrate a full recovery within 1-2 weeks (McCrory et al. 2013), the media has generally overlooked those findings and have focused instead on those cases of high profile professional athletes who require longer periods to recover. The highly publicized accounts of these high-profile cases can give the public a biased perception of recovery rates expected following a concussion. Such a bias can result in what social scientists have termed as the availability cascade, a social phenomenon where empirical data are ignored by groups in favor of information that is more available to them through examples of dramatic individual cases (Kuran and

W. B. Barr $(\bowtie)$

Departments of Neurology \& Psychiatry, NYU School of Medicine, New York, NY, USA

e-mail: william.barr@nyumc.org
Sunstein 1999). The goal of this special issue is to provide updated reviews on selective aspects of research methods that should be embraced in studies of SRC in a continuing effort to confront the ongoing availability cascade.

The first paper in this special issue covers the epidemiology of sports concussion. The authors Noble \& Hesdorffer raise the issue of whether the current level of public concern regarding SRC is the result of a true epidemic, characterized by an observed increase in its occurrence, or whether its increased recognition is the result of greater awareness and improved sensitivity of methods of assessment. The review of the acute phase of recovery by Nelson \& McCrea reaffirms the brief time course of recovery observed in most athletes following SRC and raises the question of what factors might contribute to the minority of cases that do not follow the prototypcal pattern of recovery. With a continuing search for the most effective methodology for assessing the acute effects of SRC, Register-Mihalik, Guskiewicz, \& Littleton have conducted a systematic review on the use of divided attention tasks and their utility in detecting lingering cognitive effects of the injury.

A large segment of neuropsychologists will acknowledge that the field must move towards the increased use of computerized assessment of neurocognitive functioning in both clinical and research settings. The development of sports concussion assessment methodology has helped to push the field further in that direction, although it can be argued that, to date, this has been primarily an effect of marketing rather than science. The article by Rahman-Filipiak and Woodard provides a review of the technological and environmental factors that are required for successful development and use of computerized neurocognitive testing (CNT). The article by Resch, McCrea, \& Cullum provides an update on the psychometric properties of the CNTs that are most commonly used for assessment of SRC. The two papers, in combination, provide clinicians and researchers with valuable information that will 
aid in making informed decisions on moving toward the use of CNTs for assessment of athletes following SRC or in the context of other clinical applications.

The concluding article discusses the scientific findings on long-term outcome from participation in professional contact sports and the development of dementia in retired athletes. The public is now acutely aware of cases of former National Football League (NFL) players who have died tragically and prematurely and have been found to exhibit a pattern of neuropathological abnormalities on autopsy that is reported to represent a distinct form of Chronic Traumatic Encephalopathy (CTE) (McKee et al. 2009). The review by Karantzoulis \& Randolph provides a critical reappraisal of those findings, claiming that there remains no empirical data demonstrating a definitive association between concussion in sports and increased risk for late-life cognitive and neuropsychiatric impairment, including CTE. An aim of this article and others in this special issue is to stimulate the design and execution of studies that will advance us to the next stage of acquiring objective knowledge on what has become an important public health concern and help to close the divide seen between media and evidence-based accounts of sports concussion. A common goal would be to overcome current shortcomings of all currently available SRC investigations by designing prospective longitudinal studies using quantitative assessment with high sensitivity and specificity for detection of occult to obvious SRC compromise.

Conflicts of Interest The author has no financial disclosure or conflict of interest to report.

\section{References}

Kuran, T., \& Sunstein, C. R. (1999). Availability cascades and risk regulation. Stanford Law Review, 51, 683-768.

McCrory, P., Meeuwisse, W. H., Aubry, M., Cantu, R. C., Dvorak, J., Echemendia, R. J., et al. (2013). Consensus statement on concussion in sport-the 4th International Conference on Concussion in Sport held in Zurich, November 2012. PM R, 5(4), 255-279. doi:10.1016/ j.pmrj.2013.02.012.

McKee, A. C., Cantu, R. C., Nowinski, C. J., Hedley-Whyte, E. T., Gavett, B. E., Budson, A. E., et al. (2009). Chronic traumatic encephalopathy in athletes: progressive tauopathy after repetitive head injury. Journal of Neuropathology and Experimental Neurology, 68(7), 709-735. doi:10.1097/NEN.0b013e3181a9d503. 